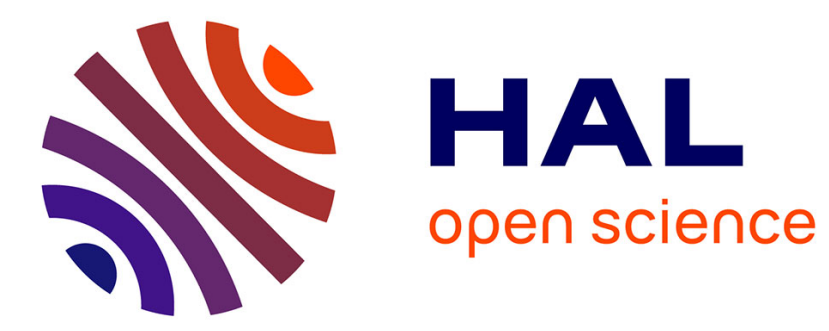

\title{
Acoustic Measurement of Surface Wave Damping by a Meniscus
}

\author{
Guillaume Michel, François Pétrélis, Stéphan Fauve
}

\section{To cite this version:}

Guillaume Michel, François Pétrélis, Stéphan Fauve. Acoustic Measurement of Surface Wave Damping by a Meniscus. Physical Review Letters, 2016, 116 (17), pp.174301 10.1103/PhysRevLett.116.174301 . hal-01493963

\section{HAL Id: hal-01493963 https://hal.sorbonne-universite.fr/hal-01493963}

Submitted on 22 Mar 2017

HAL is a multi-disciplinary open access archive for the deposit and dissemination of scientific research documents, whether they are published or not. The documents may come from teaching and research institutions in France or abroad, or from public or private research centers.
L'archive ouverte pluridisciplinaire HAL, est destinée au dépôt et à la diffusion de documents scientifiques de niveau recherche, publiés ou non, émanant des établissements d'enseignement et de recherche français ou étrangers, des laboratoires publics ou privés. 


\title{
Acoustic measurement of surface wave damping by a meniscus
}

\author{
Guillaume Michel,* François Pétrélis, and Stéphan Fauve \\ Laboratoire de Physique Statistique, École Normale Supérieure, CNRS, \\ Université P. et M. Curie, Université Paris Diderot, Paris, France
}

(Dated: March 20, 2017)

\begin{abstract}
We investigate the reflection of gravity-capillary surface waves by a plane vertical barrier. The size of the meniscus is found to strongly affect reflection: the energy of the reflected wave with a pinned contact line is around twice the one corresponding to a fully developed meniscus. To perform these measurements, a new experimental setup similar to an acousto-optic modulator is developed and offers a simple way to measure the amplitude, frequency and direction of propagation of surface waves.
\end{abstract}

PACS numbers: 43.20+g,43.58+z,47.35.Lf, 37.35.Pq,47.55.np

\section{INTRODUCTION}

Within its range of applicability, acoustics offer simple, robust and low-cost methods for performing quantitative measurements. In addition to velocimetry, the phase modulation of a harmonic signal caused by a reflection on a moving boundary can be used to track a vibrating surface with a subwavelength resolution. Motionless but corrugated surfaces can also be characterized: after the pioneer work of Rayleigh, this topic received much attention at the beginning of the 20th century, one application being the diffusion of sound at the sea surface or at the bottom of the ocean (see for instance the work of Eckart [1] or the review [2]). The study of surface waves with acoustics combines these two effects, spatially periodic deformation acting as a diffraction grating and its motion inducing a Doppler shift equal to a multiple of the frequency of the wave [3]. Apart from [3] that simply evidences this Doppler shift, it has remained a curiosity and has never been used as a measuring tool of surface waves. In contrast, an analogous effect occurs in optics when light propagates in a medium sustaining strong ultrasonic waves and is the basis of devices commonly used to shift the frequency of a laser and known as acoustooptic modulators or "Bragg cells". This analogy led us to develop a setup able to measure both the directions and amplitudes of surface waves, whose description and test are reported in the first part of this Letter.

The effect of wetting on damping of surface waves is an old but still very active topic. Theoretical studies have been carried out in a circular cylinder with several wetting boundary conditions [4-6] and are in agreement with experiments, especially in the nonwetting case $([7,8]$, and references therein). The case of damping by a pinned contact line at a nonzero height has never been investigated and is the object of the experiment described in the second part of this Letter. This is of particular interest since it provides a way to continuously change the edge condition from a pinned-end to an almost free-end. The ability to compare the amplitudes of counterpropagating waves with only a couple of piezoelectric transducers offers a great simplification to the measurement of reflection coefficients. We are then able to measure its evolution in the case of gravity-capillary waves reflecting on a plane vertical barrier whose elevation is experimentally controlled from submersion to above the liquid level. This experiment demonstrates that maximal energy reflection coefficients are obtained with a pinned contact line at the level of the unperturbed liquid and can be as much as twice the reflection coefficient corresponding to a fully developed meniscus. It provides a method for studying how this coefficient depends on the amplitude and direction of the wave or on the properties of the barrier.

\section{SURFACE WAVES MEASUREMENT USING ACOUSTICS}

Consider the situation sketched in Fig. 1 where an acoustic wave is sent with normal incidence on a perturbed water surface. The surface wave is assumed uni-

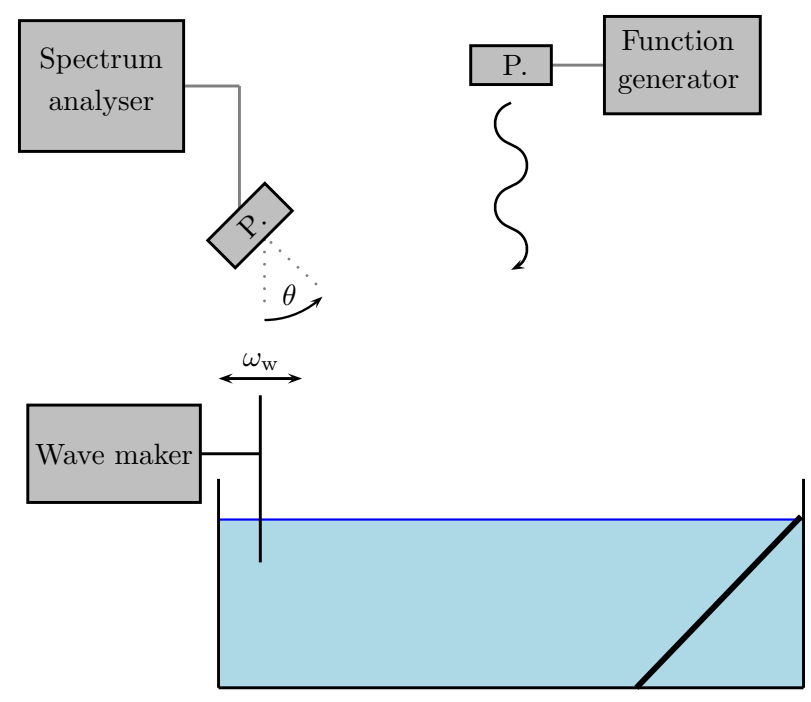

FIG. 1. Experimental setup for the measurement of surface waves. P. stands for piezoelectric transducer. 
directional, and the deformation of the free surface is denoted by $\xi(x, t)$. The wave number and angular frequency of the incident acoustic wave are $k_{0}$ and $\omega_{0}$, and the insonified area is $\Sigma$. The far field description of the scattered acoustic wave in the $\theta$ direction is then characterized by the Fraunhofer diffraction integral

$$
\int_{\Sigma} e^{i k_{0} \xi(x, t)(1+\cos (\theta))} \times e^{i k_{0} x \sin (\theta)} \mathrm{d} x
$$

where the first exponential term is the transmission function. In the case of $\xi(x, t)$ being a propagative or standing wave and apart from the geometrical correction $1+\cos (\theta)$, this integral has been worked out by Raman and Nath $[9,10]$ in their study of the first acousto-optic modulators made by Debye [11] and Lucas [12]. In order to use these results, we shall stay in the limit $k_{0} \xi \ll 1$ in which (1) becomes linear in $\xi$. Up to the first order in this parameter and taking into account the correction $1+\cos (\theta)$, the results of $[9,10]$ for a propagative surface wave $\xi(x, t)=\xi_{0} \cos \left(\omega_{\mathrm{w}} t \pm k_{\mathrm{w}} x\right)$ can be summed up as follows:

1. Two diffracted beams centered on directions given by $\sin (\theta)= \pm k_{\mathrm{w}} / k_{0}$ are observed.

2. Their relative intensity, compared to the one of the backscattered components, is

$$
\frac{I_{ \pm 1}}{I_{0}}=\left(\frac{\left(1+\sqrt{1-\left(k_{\mathrm{w}} / k_{0}\right)^{2}}\right) k_{0} \xi_{0}}{2}\right)^{2}
$$

3. The beam diffracted in the direction of propagation of the surface wave has an angular frequency $\omega_{0}+$ $\omega_{\mathrm{w}}$.

4. The beam diffracted in the opposite direction has an angular frequency $\omega_{0}-\omega_{\mathrm{w}}$.

In this linear regime, the scattered signal received by the measurement transducer in Fig. 1 is then a sum of harmonic signals whose amplitudes and frequencies are directly related to the ones of the water waves and whose Doppler shift signs can distinguish between counterpropagating waves.

The following setup is used to test these results: a basin of dimension $648 \times 846 \times 160 \mathrm{~mm}$ is filled with water, an oblique plate being fixed at one extremity to limit reflections of water waves. At the other side, a Brüel \& Kjær 4810 shaker drives a vertical plate sinusoidally at an angular frequency $\omega_{\mathrm{w}}=2 \pi f_{\mathrm{w}}$. Piezoelectric transducers resonant at $f_{0}=41500 \mathrm{~Hz}\left(\omega_{0}=2 \pi f_{0}\right)$ emit and receive at $\theta=45.2^{\circ}$ the acoustic signal, then processed by a HP $35670 \mathrm{~A}$ spectrum analyser. A position sensing detector tracks the motion amplitude of a laser beam after its reflection on the surface: this part of the setup, not shown in Fig. 1, is used to measure the amplitude of propagative water waves when no reflection is observed.
Propagative gravity-capillary waves of frequency $f_{\mathrm{w}}=$ $15 \mathrm{~Hz}$, corresponding to $k_{\mathrm{w}} \simeq 400 \mathrm{~m}^{-1}$, are generated, no noticeable reflected waves being observed. All this study is done in the limit of small $k_{0} \xi$ and $k_{\mathrm{w}} \xi$, in order to stay in the linear regime for both acoustic scattering and water wave propagation. The received scattered spectra reported in Fig. 2 consist of one component at $f_{0}$ caused by multiple reflections and a second one, Doppler shifted, at $f_{0}-f_{\mathrm{w}}$. The amplitude of this latter component is found linear in $\xi_{0}$, see Fig. 3. It is also checked (figures not reported here for brevity) that reversing the direction of the propagative waves changes the sign of the Doppler shift, and that the acoustic amplitude spectra are linear in $\xi(x, t)$.

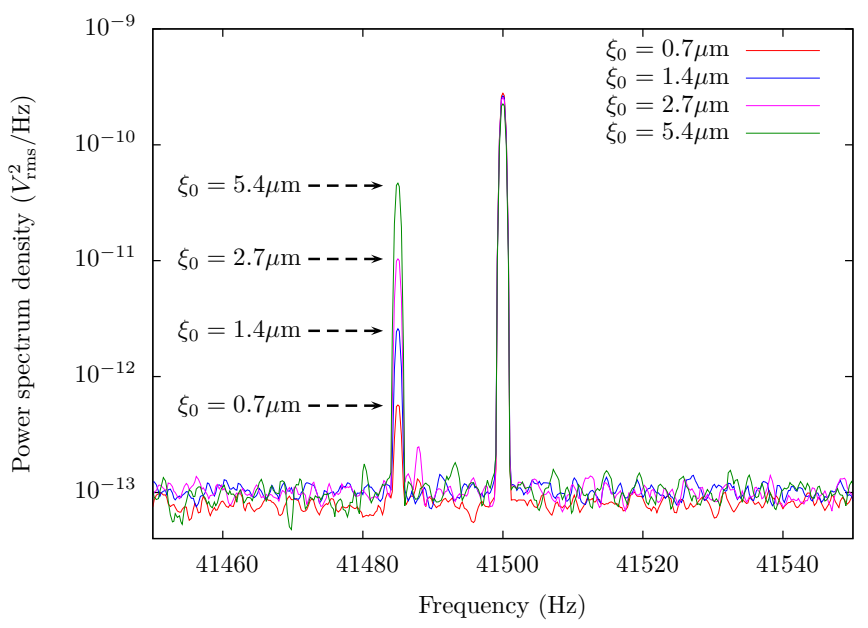

FIG. 2. Acoustic spectra for different surface wave amplitudes: only one Doppler shifted component is present. If $k_{0} \xi$ is not small compared to 1 , other harmonics can be observed.

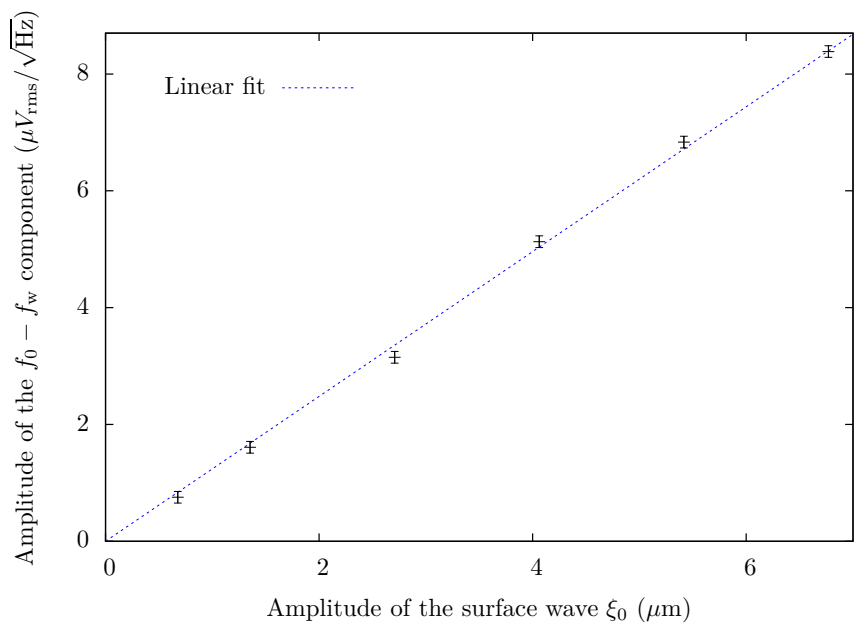

FIG. 3. Linear growth of the Doppler-shifted component with the amplitude of the surface waves. 
These results are in agreement with items 2 to 4 of the above list. In this experiment, some signal is received even if $\arcsin \left(k_{\mathrm{w}} / k_{0}\right) \simeq 32^{\circ} \neq \theta$, showing that the angular diffraction window is wide, i.e., that a large range of water wave frequencies can be detected without changing the angle of the receiver. As for usual diffraction gratings, this window is expected to become smaller as the insonified area increases. We also note that the Doppler shift is a key factor in this experiment, making scattering distinguishable from specular reflections. Both this background noise and the angular window make the details of (2) irrelevant: from an experimental point of view, it reduces to $I(\theta)=\alpha\left(\theta, k_{\mathrm{w}}\right) \xi_{0}^{2}$, where the calibration function $\alpha\left(\theta, k_{\mathrm{w}}\right)$ has to be measured or removed by comparing two counterpropagating waves as is done in the next section.

\section{MENISCUS DAMPING}

The previous setup is slightly changed to measure reflection coefficients of gravity-capillary waves: a vertical barrier consisting of a $2.9 \mathrm{~mm}$-thick dural plate of width $8 \mathrm{~cm}$ is fixed $30 \mathrm{~cm}$ away from the wave maker. Its height $h$ can be set and measured with a $0.1 \mathrm{~mm}$ precision. The top matches the unperturbed water level if $h=0$ (see Fig. 4).

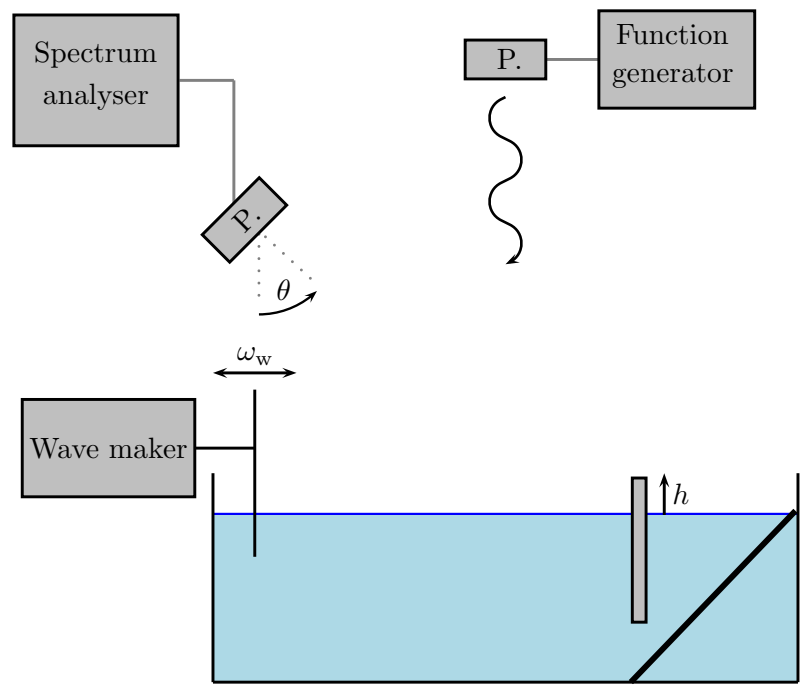

FIG. 4. Experimental setup for the measurement of the reflection coefficient. P. stands for piezoelectric transducer and the distance between the vertical barrier and the edge of the basin is longer than sketched here.

In the steady-state, two counterpropagating water waves of frequency $f_{\mathrm{w}}$ cross the insonified area:

- one of amplitude $\xi_{0}$ going toward the barrier, leading to a received acoustic signal of frequency $f_{0}-f_{\mathrm{w}}$ and intensity $\alpha\left(\theta, k_{\mathrm{w}}\right) \xi_{0}^{2}$.
- Another of amplitude $R_{\text {tot }} \xi_{0}$ moving away from the barrier, $R_{\text {tot }}$ standing for a total reflection coefficient. The scattered acoustical signal received by the piezoelectric transducer is of frequency $f_{0}+f_{\mathrm{w}}$ and intensity $\alpha\left(\theta, k_{\mathrm{w}}\right)\left(R_{\mathrm{tot}} \xi_{0}\right)^{2}$.

The coefficient $R_{\text {tot }}^{2}$ can then be easily measured with a spectrum analyser by dividing the power spectral density at $f_{0}+f_{\mathrm{w}}$ by the one at $f_{0}-f_{\mathrm{w}}$. Typical spectra are displayed in Fig. 5, showing a strong dependence of $R_{\text {tot }}$ on $h$. Given that the wavelength of the incident surface wave $\left(2 \pi / k_{\mathrm{w}} \simeq 1.6 \mathrm{~cm}\right)$ is small compared to the width of the plate and the depth of water, this experiment models the reflection of gravity-capillary waves on an infinite vertical barrier in deep water. In all these experiments, $\xi_{0}$ is maintained small compared to the wavelength of the acoustic wave $\left(k_{0} \xi_{0} \ll 1\right)$ and of the water wave $\left(k_{\mathrm{w}} \xi_{0} \ll 1\right)$ : $R_{\text {tot }}$ is found independent of the water wave steepness $k_{\mathrm{w}} \xi_{0}$ in the explored ranged $\left(k_{\mathrm{w}} \xi_{0}\right.$ up to $0.05)$. In this linear propagation regime, the total reflection coefficient $R_{\text {tot }}$ writes

$$
R_{\text {tot }}(h)^{2}=D(h)^{2} \times R(h)^{2},
$$

where $D(h)$ stands for the linear damping during the propagation and $R(h)^{2}$ for the energy reflection coefficient at the barrier. The dependence of $D(h)$ on $h$, resulting from the variation of the free-surface area of the meniscus, will be considered as weak and neglected. Moreover, the possible contamination of the interface by surface-active agents makes $D(h)$ difficult to deduce from theory. Therefore, we consider a parameter describing only reflection,

$$
\left(\frac{R_{\mathrm{tot}}(h)}{R_{\mathrm{tot}}(\infty)}\right)^{2} \simeq\left(\frac{R(h)}{R(\infty)}\right)^{2},
$$

which compares the efficiency of reflection when the meniscus is of height $h$ to the case of a fully developed

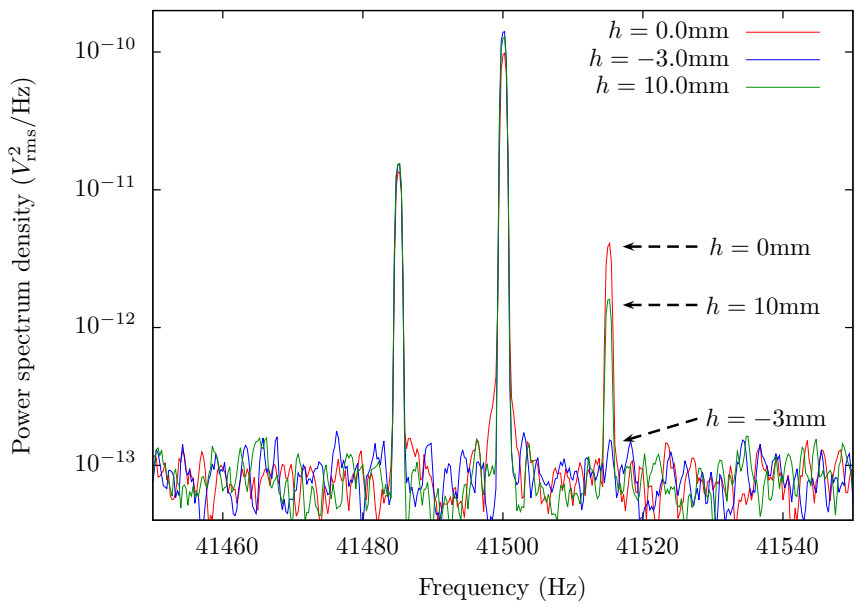

FIG. 5. Typical acoustic spectra for different elevations. 
meniscus. Its dependence on $h$ is reported in Fig. 6. We comment on the different branches of this graph:

- the fully immersed barrier is obtained for values of $h$ smaller than $-1 \mathrm{~mm}$. This regime cannot be extended to $0>h \geqslant-1 \mathrm{~mm}$ as a dewetting transition is always observed. Because of the strong localization of the energy of a surface wave close to the interface (the velocity field decays exponentially with a penetration length $1 / k_{\mathrm{w}} \simeq 2.5 \mathrm{~mm}$ ), the reflection coefficient can hardly be distinguished from zero. This result has been predicted for gravity waves [13] and generalized to gravity-capillary waves [14]. Theoretical values of $R(h)^{2}$ using equations in section 6 of [14] for $h=-2 \mathrm{~mm}$ and $h=-1 \mathrm{~mm}$ are, respectively, 0.05 and 0.21 (note that Fig. 6 shows $(R(h) / R(\infty))^{2}$ and not $\left.R(h)^{2}\right)$.

- At heights $h>5 \mathrm{~mm}$, the reflection coefficient is nearly constant. The meniscus is fully developed and the contact line no longer pinned at the top corner of the barrier, its motion giving rise to an additional dissipation. The theoretical investigation of this problem is complex since the usual noslip boundary condition (BC) leads to a divergence. Other BCs have to be considered, involving an additional length scale such as a slip length or a microscopic cut off [15]. Even though the first observations are almost a century old [16], a global picture of the local dissipation close to the contact line is still missing and has only been achieved in a few limits (see $[17,18]$ and references therein). Using such a $\mathrm{BC}$, the reflection coefficient $R(\infty)^{2}$ in the limit of small amplitudes and inviscid fluid can be expressed as a function of a phenomenological parameter [19].

- Between these two limiting cases, the contact line is pinned at the edge of the barrier, the meniscus being of height $h$. The value of the contact angle of the unperturbed surface is not a free parameter but can be deduced from $h$. The maximum reflection coefficient is observed when the meniscus vanishes, that is $h=0$.

Within the precision of our measurements, no discontinuity occurs when the contact line leaves the corner ( $h \simeq 5 \mathrm{~mm}$ ), showing that the edge condition continuously changes from a pinned-end one to an almost freeend one, which can be described using the phenomenological law reported in [19] and commonly used in the literature.

\section{CONCLUSION}

We have shown how acoustic waves can be used to characterize surface waves. We emphasize that this

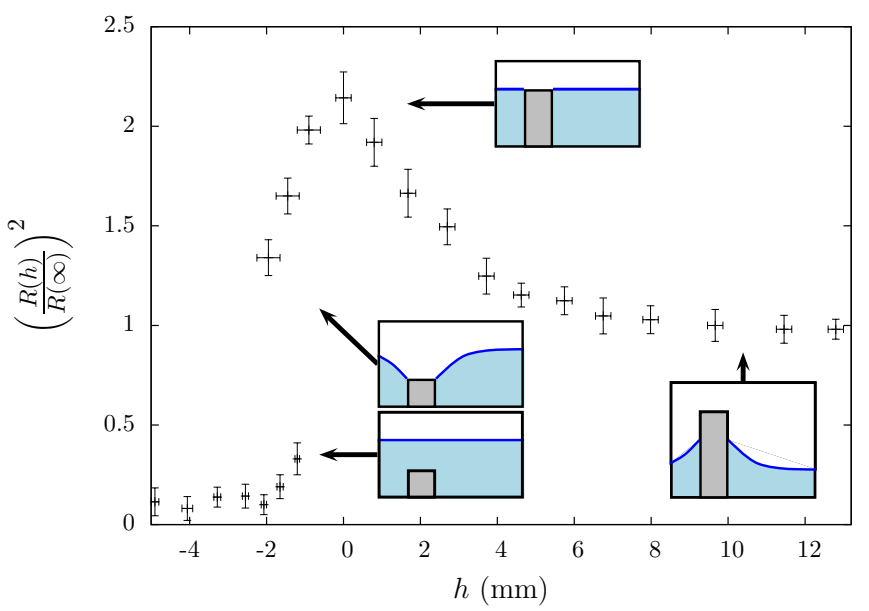

FIG. 6. Evolution of the normalized energy reflection coefficient as a function of the height of the barrier $h$.

method gives access to both amplitudes of counterpropagating waves. Even if the focus was set on water waves, the same setup can be applied to any kind of surface waves, as long as its amplitude and frequency remain small compared to the acoustical ones. Other potential candidates include Rayleigh waves or dynamical deformations of thin metallic plates, films or membranes, in which the full deformation field may often be acquired with optics but with a much more complex and expensive setup.

This method was then used to measure the reflection coefficient of gravity-capillary waves depending on the size of the meniscus. We report a strong dependence on this parameter: between a pinned contact line with no meniscus and a fully developed meniscus with no specific constraint on the contact line, the energy reflection coefficient may be divided by as much as two. The present work about wetting is a glimpse into a vast domain and could, for instance, be extended to the study of nonlinearities in the case of high amplitude waves. The dependence of this effect on the frequency and direction of the surface wave and on the properties of the barrier (which could be nonvertical, hydrophobic, porous, or nonhomogeneous) is also of interest. The reflection process also leads to a time shift which has been predicted in some limits (see, e.g., [20]) and whose dependence can be measured with this setup.

This work is supported by CNES and ANR-12-BS040005-02.

email: guillaume.michel@ens.fr

[1] C. Eckart, J. Acoust. Soc. Am. 25, 566 (1953).

[2] L. Fortuin, J. Acoust. Soc. Am. 47, 1209 (1970).

[3] L. N. Liebermann, J. Acoust. Soc. Am. 35, 923 (1963). 
[4] J. W. Miles, Proc. R. Soc. A 287, 459 (1967).

[5] J. Grahamn-Eagle, Math. Proc. Cambridge Philos. Soc. 94, 553 (1983).

[6] L. M. Hocking, J. Fluid Mech. 179, 253 (1987).

[7] B. Cocciaro, S. Faetti and M. Nobili, J. Fluid Mech. 231, 325 (1991).

[8] B. Cocciaro, S. Faetti and C. Festa, J. Fluid Mech. 246 , 43 (1993).

[9] C. V. Raman and N. S. Nagendra Nath, Proc. Indian Acad. Sci. 2, 406 (1935).

[10] C. V. Raman and N. S. Nagendra Nath, Proc. Indian Acad. Sci. 3, 75 (1936).

[11] P. Debye and F. W. Sears, Proc. Nat. Acad. Sci. U.S.A. 18, 409 (1932).

[12] R. Lucas and P. Biquard, C. R. Acad. Sci. Paris 194, $2132(1932)$.
[13] W. R. Dean, Math. Proc. Cambridge Philos. Soc. 41, 231 (1945).

[14] D. V. Evans, Math. Proc. Cambridge Philos. Soc. 64, 795 (1968).

[15] P. G. de Gennes, Rev. Mod. Phys. 57, 827 (1985).

[16] R. Ablett, Philos. Mag. 46, 244 (1923)

[17] C.-L. Ting and M. Perlin, J. Fluid Mech. 295, 263 (1995).

[18] S. Guo, H. Lee, P. Sheng and P. Tong, Phys. Rev. E 91, 012404 (2015).

[19] P. F. Rhodes-Robinson, Math. Proc. Cambridge Philos. Soc. 92, 369 (1982).

[20] J. M. Perez-Gracia, J. Porter, F. Varas and J. M. Vega, J. Fluid Mech. 739, 196 (2014). 